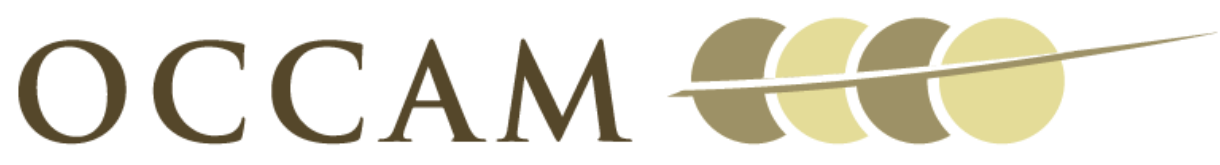

OXFORD CENTRE FOR COLlaborative APPLIED MATHEMATICS

Report Number 09/11

A Comparison of Numerical Methods used for

Finite Element Modelling of Soft Tissue

Deformation

by

P. Pathmanathan, D. J. Gavaghan, J. P.

Whiteley

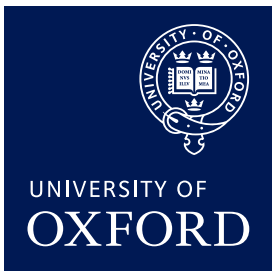

Oxford Centre for Collaborative Applied Mathematics Mathematical Institute 24 - 29 St Giles'

Oxford

OX1 3LB

England 



\title{
A Comparison of Numerical Methods used for Finite Element Modelling of Soft Tissue Deformation
}

\author{
P. Pathmanathan, D. J. Gavaghan, J. P. Whiteley
}

February 27, 2009

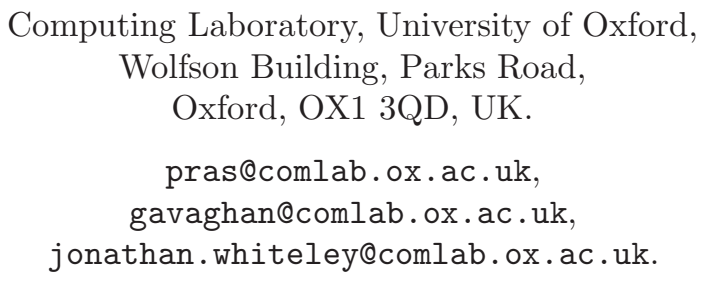

\begin{abstract}
Soft tissue deformation is often modelled using incompressible nonlinear elasticity, with solutions computed using the finite element method. There are a range of options available when using the finite element method, in particular, the polynomial degree of the basis functions used for interpolating position and pressure, and the type of element making up the mesh. We investigate the effect of these choices on the accuracy of the computed solution, using a selection of model problems motivated by typical deformations seen in soft tissue modelling. We set up model problems with discontinuous material properties (as is the case for the breast), steeply changing gradients in the body force (as found in con-
\end{abstract}


tracting cardiac tissue), and discontinuous first derivatives in the solution

at the boundary, caused by a discontinuous applied force (as in the breast during mammography). We find that the choice of pressure basis functions are vital in the presence of a material interface, higher-order schemes do not perform as well as may be expected when there are sharp gradients, and in general that it is important to take the expected regularity of the solution into account when choosing a numerical scheme.

\section{Keywords: Finite element method, nonlinear elasticity, basis func- tions, breast, heart.}

\section{Introduction}

Simulating soft tissue deformation is a rapidly developing field in computational biology. Although linear elasticity is sometimes used in such models $[1,2,3]$, the strains exhibited by soft tissues are typically of magnitudes which necessitate the use of the more complex, nonlinear, theory of elasticity $[4,5]$. Two organs which have received particular attention using nonlinear elasticity are the breast and the heart. The breast shape varies significantly between different clinical imaging situations - for example, hanging under gravity during magnetic resonance imaging, compared to heavily compressed during mammography (X-ray imaging) - and a model of the deformation undergone by the breast can be used for image-based guidance or to aid image-based diagnosis $[6,7,8]$. Computational modelling of the electro-mechanical behaviour of the heart is an important tool in investigating normal and aberrant cardiac function $[9,10,11]$. Such models are typically extremely complex, coupling cell-level models of electrical excitation and active tension generation with tissue-level models of electrical conductance and mechanical deformation. The deformation of various other 


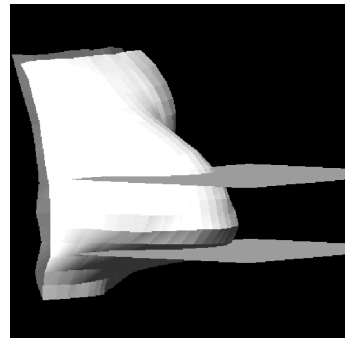

(a)

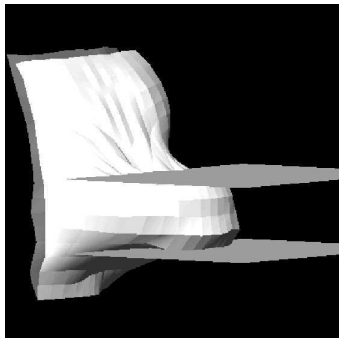

(b)

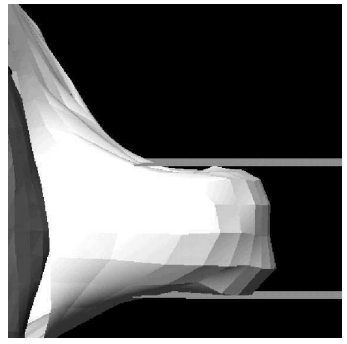

(c)

Figure 1: Simulating the compression of the breast during mammography. Note that only front (skin, lighter coloured) and back (pectoral muscle, darker coloured) surfaces of the breast are shown. Also shown are the compression plates. (a) The initial state before compression. (b) The computed compressed breast. (c) Alternative view of the compressed state.

tissues or organs have also been studied using nonlinear elasticity, including, among others, skeletal muscle [12], ligaments [13], cartilage [14], the liver [15], mesentery [16], blood vessels [17] and tumour spheroids [18].

The finite element (FE) method [19] is commonly used to compute numerical solutions of the partial differential equations which govern such soft tissue deformation. As an illustration of the use of nonlinear elasticity and the FE method in soft-tissue modelling, and motivation for the results in this paper, a three-dimensional simulation of the deformation of the breast is presented in Figure 1. The mesh of the breast is constructed from magnetic resonance images, and is shown in Figure 1(a), together with the location of two compression plates that would be used to compress the breast during mammography. The mesh is comprised of hexahedral elements, linear basis functions are used for displacement and piecewise constant basis functions for pressure (terms which will be introduced below). Figures 1(b) and (c) show the deformed breast, from two viewpoints, found by solving the contact problem with nonlinear elasticity, assuming zero body force and surface tractions, and zero displacement on the pectoral muscle. For further details of this model, see [6]. 
Within the FE method, unknowns are approximated using piecewise polynomial basis functions defined over some (typically tetrahedral or hexahedral) tessellation of the solution domain ${ }^{1}$. There are then a number of decisions that must be made when implementing the FE method, and a range of approaches have been adopted by the authors cited above. One such choice is in the order of the polynomial basis functions used for approximating the unknown displacement, with common choices being piecewise linear or piecewise quadratic basis functions. The latter quadratically interpolate an unknown over an element but require the element to have extra nodes in addition to the vertices, and the numerical solution to be computed at these extra nodes (see Figure 2). An alternative choice is to use cubic-Hermite bases - these interpolate the displacement, the first spatial derivatives of the displacement, and, in two-dimensions, the second spatial cross-derivative [20], from their nodal values (see Figure 2). Therefore, if cubic-Hermite bases are used, these derivatives have to be computed at each node, in addition to the displacement itself, greatly increasing the computation time, but with the intention of increasing accuracy. For more details on the definitions of these basis functions see [19] and [20].

In incompressible elasticity the constraint of incompressibility is enforced by introducing a Lagrange multiplier, which can be interpreted as the internal pressure, and which also has to be computed. A second choice in FE implementation is therefore the order of polynomial interpolation used for this pressure. It has been shown that the order of interpolation for position must be higher than that used for the pressure [21], as otherwise the method fails to satisfy the Ladyzhenskaya-Babuska-Brezzi (LBB) 'inf-sup' condition [22] and numerical solutions may not converge to the exact solution as the stepsize tends to zero.

\footnotetext{
${ }^{1}$ The terminology shape function is often used and can be considered loosely synomynous with 'basis function'. Shape functions are basis functions restricted to a single (canonical) element, while basis functions are functions on physical space composed of all shape functions which correspond to a given node.
} 
Suitable choices of basis function for interpolating pressure, given the choices described above for displacement, are therefore: piecewise constant (functions which take the value 1 in one element and 0 in all others), piecewise linear, or piecewise quadratic bases. Another choice that is available is in the type of element used in the FE mesh, tetrahedral or hexahedral (or their counterparts in 2D). These elements have been seen to sometimes give different numerical results in solid mechanics problems $[23,24]$. Furthermore, linear basis functions cannot be used with triangular/tetrahedral elements without introducing significant numerical error in the solution (see [25], which explains that triangular/tetrahedral elements for displacement and piecewise constant elements for pressure cannot be used for the Stokes' Problem. The Stokes' Problem is formally identical to incompressible linear elasticity with pressure, and therefore this combination of elements should not be used for linear elasticity, and hence not for nonlinear elasticity either).

To our knowledge, there has been no systematic comparison of the relative accuracy of these differing approaches to the implementation of the FE method in modelling the deformation of soft tissue. In this paper we therefore study the numerical behaviour and computational efficiency of a representative selection of different methods, on a range of $2 \mathrm{D}$ model problems designed to replicate the type of behaviour which arise in modelling soft tissue. We investigate the following four choices of numerical scheme: (a) linear (tensor-product) bases for position and piecewise constant bases for pressure, on quadrilateral elements (as used in biomechanical modelling in $[6,10,26]$ ), which we refer to as the linear scheme; (b) quadratic bases for position and linear bases for pressure, on triangular elements (as used in biomechanical modelling in [27, 28]), referred to as the quad-tri scheme; (c) quadratic (tensor-product) bases for position and linear (tensor-product) bases for pressure, on quadrilateral elements (used 
in biomechanical modelling in $[29,30])$, referred to as the quad-quad scheme; and (d) cubic-Hermite (tensor-product) bases for position and linear (tensorproduct) bases for pressure, on quadrilateral elements ([11, 31, 32]), referred to as the cubic-Hermite scheme. These options are summarised in Table 1, with the elements required for the four schemes illustrated in Figure 2.

We use four model problems, three of which are constructed so that their solution is known analytically, which means that, for each of the numerical methods, the method's error and therefore convergence properties (in spatial stepsize and number of degrees of freedom) can be studied. To avoid the high computational cost of performing convergence analyses in 3D, we mostly use two-dimensional plane-strain problems. The first model problem involves a simple smooth nonlinear deformation, and has two forms, one in 2D and one in 3D, referred to as Model Problems 1a and 1b. The 3D version is investigated in order to gain an insight into how the conclusions for the other 2D model problems will carry over to three-dimensions. We will also use the 3D model problem to investigate the computational cost of each of the methods. The remaining model problems are set up in two dimensions. In Model Problem 2, the elastic body is assumed have discontinuous material properties, as is the case for the breast (where there is an inner fibroglandular region covered by an outer fatty region), and can be the case for other tissues if a high spatial resolution is used. The heterogeneity is modelled by taking different material parameters for different elements in the mesh, as has regularly been carried out in computational models of the breast $[6,7,8]$. In the third model problem, the solution deformation has a steep gradient, which means the specified body force has a very steep gradient. This is designed to mimic the deformations which arise in cardiac tissue, where a steep wavefront of electrical excitation propagating across the tissue gives rise to steep variations in the generated stress. The fourth model problem is moti- 
vated by the compression of the breast during mammography. In this problem, for which the exact solution is not known analytically, we apply a discontinuous surface traction boundary condition to the top surface of the elastic body.

We begin in Section 2.1 by introducing the equations of nonlinear elasticity, and describing the FE solution procedure. The four model problems are then defined in Section 2.2. The convergence results of the four schemes on the four model problems are presented in Section 3, and, finally, we discuss the results in Section 4.
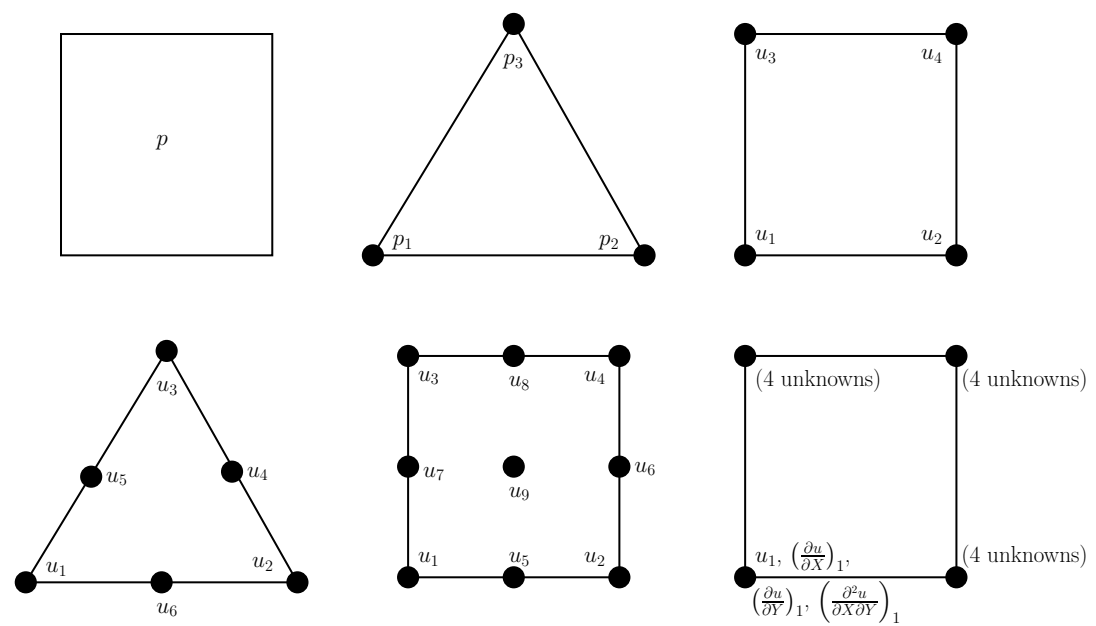

Figure 2: The elements used in the numerical schemes, together with the number of unknowns which have to be computed, illustrated using either the pressure, $p$, or the displacement in the $X$-direction, $u$. Note that different combinations of these 6 elements are used in each of the 4 schemes (so that the Linear method would use the top-right element for $u$ and for $v$ (the two components of displacement), and the top-left element for $p$ ). Top row: piecewise constant quadrilateral (used in the linear scheme); linear triangle (used for the pressure in the quad-tri scheme); (bi-)linear quadrilateral (used for displacement in the linear scheme and pressure in the quad-quad and cubic-Hermite schemes). Bottom row: quadratic triangle (used for displacement in the quad-tri scheme); (bi-)quadratic quadrilateral (used for displacement in the quad-quad scheme); cubic-Hermite quadrilateral (where the given derivatives of the unknown also have to be computed at each node). 


\begin{tabular}{ccccc} 
Name & Basis deg. for $\boldsymbol{x}$ & Basis deg. for $p$ & Element & Other \\
\hline Linear & 1 & 0 & Quadrilateral & \\
Quad-tri & 2 & 1 & Triangular & \\
Quad-quad & 2 & 1 & Quadrilateral & \\
Cubic-Hermite & 3 & 1 & Quadrilateral & Also solve for \\
& & & & derivs of $\boldsymbol{x}$
\end{tabular}

Table 1: Numerical finite element schemes to be compared.

\section{Methods}

\subsection{Nonlinear elasticity}

Let the region occupied by the undeformed elastic body be denoted $\Omega_{0} \subset \mathbb{R}^{N}$ ( $N=2$ or 3 ), and the corresponding region occupied by the deformed body be denoted $\Omega$. Given a point $\boldsymbol{X} \in \Omega_{0}$, let the corresponding deformed position be $\boldsymbol{x} \equiv \boldsymbol{x}(\boldsymbol{X}) \in \Omega$. The displacement is then given by $\boldsymbol{u}=\boldsymbol{x}-\boldsymbol{X}$. The deformation gradient is defined as $F_{M}^{i}=\frac{\partial x^{i}}{\partial X^{M}}$. The right Cauchy-Green deformation tensor is defined as $\mathbf{C}=\mathbf{F}^{\mathrm{T}} \mathbf{F}$, and the Lagrangian strain as $\mathbf{E}=\frac{1}{2}(\mathbf{C}-\mathbf{I})$, where I is the identity matrix. We will use two measures of stress: the 1st PiolaKirchhoff stress, S, which represents the force measured per unit undeformed area acting on the deformed body, and the 2 nd Piola-Kirchhoff stress, $\mathbf{T}=\mathbf{S F}^{-\mathrm{T}}$, defined to represent the force measured per unit undeformed area acting on a surface in the undeformed body [33]. We assume hyperelasticity, for which a material-dependent strain energy function, $W(\mathbf{E})$, relates stress and strain through $T^{M N}=\partial W / \partial E_{M N}$.

For incompressible materials the constraint $\operatorname{det} \mathbf{F}=1$ has to be imposed. This constraint is enforced using a Lagrange multiplier $p(\boldsymbol{X})$, which plays a similar role to the pressure in fluid flow equations. In this case, $W$ takes the form $W(\mathbf{C})=\bar{W}(\mathbf{C})-\frac{p}{2}(\operatorname{det} \mathbf{C}-1)$, where $\bar{W}$ is the material-dependent part of the strain energy [31]. In the numerical simulations in this paper, we choose 
the Neo-Hookean strain energy [34], which, restricted to two-dimensions, is

$$
W(\mathbf{C})=c(\operatorname{tr} \mathbf{C}-2)-\frac{p}{2}(\operatorname{det} \mathbf{C}-1),
$$

where $c>0$ is a material parameter. Then $\mathbf{T}=2 c \mathbf{I}-p \mathbf{C}^{-1}$, and so

$$
\mathbf{S}=2 c \mathbf{F}^{\mathrm{T}}-p \mathbf{F}^{-1}
$$

The equations governing the static equilibrium of an incompressible nonlinear elastic body, under body force $\mathbf{b}$, are [33]

$$
\begin{aligned}
\frac{\partial S^{M i}}{\partial X^{M}}+\rho_{0} b^{i} & =0, \\
\operatorname{det} \mathbf{F} & =1,
\end{aligned}
$$

(where $\rho_{0}$ is the density in the undeformed body), subject to the boundary conditions

$$
\begin{array}{rlr}
\boldsymbol{u} & =\boldsymbol{u}^{*} & \text { on } \partial \Omega_{0}^{\text {disp }}, \\
\mathbf{S}^{\mathrm{T}} \boldsymbol{N} & =\boldsymbol{s} & \text { on } \partial \Omega_{0}^{\text {trac }},
\end{array}
$$

where $\boldsymbol{u}^{*}$ is a specified displacement, $s$ is a specified surface traction, $\boldsymbol{N}$ in the outward-facing unit normal on the boundary of the undeformed body, and $\partial \Omega_{0}^{\text {disp }}$ and $\partial \Omega_{0}^{\text {trac }}$ partition the boundary of the undeformed body.

To solve this system using the finite element method, (3) and (4) are first converted into the equivalent weak formulation, by multiplying them respec- 
tively by test functions $\boldsymbol{v}$ and $q$, summing, and integrating by parts, to obtain

$$
\begin{aligned}
0= & \int_{\Omega_{0}} S^{M i} \frac{\partial v_{i}}{\partial X^{M}} \mathrm{~d} V_{0}-\int_{\Omega_{0}} \rho_{0} b^{i} v_{i} \mathrm{~d} V_{0} \\
& -\int_{\partial \Omega_{0}^{\text {trac }}} s^{i} v_{i} \mathrm{~d} S_{0}+\int_{\Omega_{0}} q(\operatorname{det} \mathbf{F}-1) \mathrm{d} V_{0} \quad \forall \boldsymbol{v} \in \mathcal{V}, q \in \mathcal{Q},
\end{aligned}
$$

where $\mathcal{V}$ is (loosely-speaking) the set of differentiable functions on $\Omega_{0}$ that are zero on $\partial \Omega_{0}^{\text {disp }}$, and $\mathcal{Q}$ is the set of square-integrable functions on $\Omega_{0}$. The domain $\Omega_{0}$ is then discretised into a mesh comprising of nodes and (in 2D) quadrilateral or triangular elements. Basis functions are next chosen to interpolate the displacement and pressure given their nodal values. $\boldsymbol{v}$ and $q$ are then allowed to vary only in the finite-dimensional spaces spanned by the appropriate choice of basis functions, which converts (7) into a finite set of nonlinear equations [35]. We solve this nonlinear system using a total Lagrangian approach and Newton's method (with a simple line search in the Newton direction where, if the computed Newton update is a, we test updates of $\lambda \mathbf{a}$ for several (of the order of ten) values of $\lambda \in(0,1]$ to see which update most decreases the residual of the nonlinear system) [35]. The linear systems that arise from use of the Newton method are solved using the restarted GMRES (Generalised Mimimal Residual) method [36], an iterative Krylov subspace method for computing the numerical solution of large non-symmetric linear systems. This was implemented using $\mathrm{C}$ and $\mathrm{C}++$.

\subsection{Model Problems}

We now describe the set of four model problems which we construct in order to investigate the accuracy and convergence behaviour of the schemes discussed above. Each of the model problems are specified by a definition of the body force, $\boldsymbol{b}(\boldsymbol{X})$, and a set of boundary conditions, i.e. a definition of $\boldsymbol{u}^{*}, \boldsymbol{s}, \partial \Omega_{0}^{\mathrm{disp}}$ 
and $\partial \Omega_{0}^{\text {trac }}$, and each of Model Problems 1-3 is constructed so that the solution displacement and pressure are known analytically. All model problems are constructed in two dimensions taking $\Omega_{0}$ as the unit square, with the exception of Model Problem 1b which is defined in $3 \mathrm{D}$ with $\Omega_{0}$ taken to be the unit cube, and using the Neo-Hookean material law (1). The body is heterogeneous in the second problem, and homogeneous in the others. As stated above, all model problems except the first are motivated by the types of deformation encountered in soft-tissue modelling. The four model problems are illustrated in Figure 3.

\subsubsection{Model Problem 1: A smooth nonlinear deformation}

\section{Model Problem 1a (2D)}

Let us fix the left-hand edge of the square: $\boldsymbol{u}^{*}=0$ on $X=0$, and choose as the solution deformation

$$
\boldsymbol{x}=\left(\begin{array}{c}
X+a X^{2} / 2 \\
Y(1+a X)^{-1}
\end{array}\right)
$$

for some $a>0$, representing stretching in the $X$-direction and irregular compression in the $Y$-direction. This deformation is illustrated in Figure 3(a). Now,

$$
\mathbf{F}=\left(\begin{array}{cc}
1+a X & 0 \\
-Y a(1+a X)^{-2} & (1+a X)^{-1}
\end{array}\right)
$$

has determinant one as required. Choosing $p=2 c$, and defining $\lambda=1+a X$, the 1st Piola-Kirchhoff stress is then, using (2),

$$
\mathbf{S}=2 c\left(\begin{array}{cc}
\lambda-\lambda^{-1} & -Y a \lambda^{-2} \\
-Y a \lambda^{-2} & \lambda^{-1}-\lambda
\end{array}\right) .
$$

We can then calculate the body force and surface tractions on the remaining 


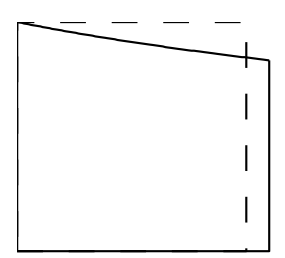

(a)

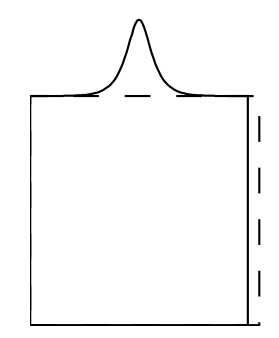

(c)
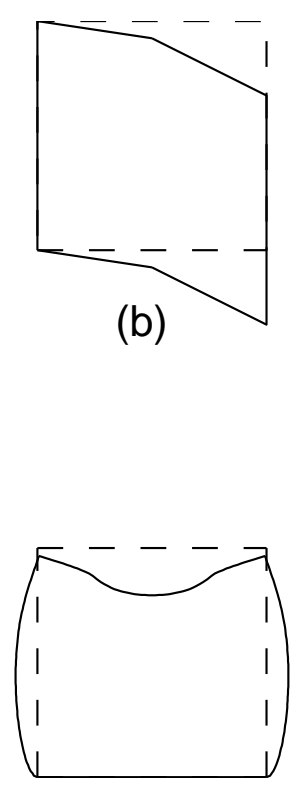

(d)

Figure 3: Deformed shapes for each of the model problems (solid lines), with undeformed unit square (dashed lines). (a): Model Problem 1a, where the body is stretched in the $X$-direction and compressed in the $Y$-direction (more stretching/compression as $X$ increases). Model Problem $1 \mathrm{~b}$ is a $3 \mathrm{D}$ variation on this. (b): Model Problem 2, where the body has discontinuous material properties and the solution is one shear with gradient $-\alpha$ in $X \leq 0.5$, and a second shear with gradient $-\beta$ in $X>0.5$. (c): Model Problem 3, where the solution is near zero displacement for $X<0.5$, a sharp compression in the $X$-direction (hence elongation in the $Y$-direction) around $X=0.5$, and a translation inwards for $X>0.5$. (d): Model Problem 4, where the bottom surface is fixed, and a discontinuous force is applied to the top surface: zero traction except in the region $\{X \in[0.25,0.75], Y=1\}$. Note that in Model Problems $1-3$, only the left-hand edge is fixed, surface tractions are applied to the remaining three edges. 
edges required to produce the deformation (8), using (3) and (6):

$$
\begin{aligned}
& \boldsymbol{b}=\frac{-2 c}{\rho_{0}}\left(\begin{array}{c}
a \\
2 Y a^{2} \lambda^{-3}
\end{array}\right), \\
& s= \begin{cases}2 c\left(\begin{array}{c}
0 \\
\lambda-\lambda^{-1}
\end{array}\right) & \text { if } Y=0, \\
2 c\left(\begin{array}{c}
-a \lambda^{-2} \\
\lambda^{-1}-\lambda
\end{array}\right) & \text { if } Y=1, \\
2 c\left(\begin{array}{c}
\lambda-\lambda^{-1} \\
-Y a \lambda^{-2}
\end{array}\right) & \text { if } X=1 .\end{cases}
\end{aligned}
$$

\section{Model Problem 1b (3D)}

For a $3 \mathrm{D}$ variation on Model Problem 1a, let $\Omega_{0}$ be the unit cube, and let us define

$$
\boldsymbol{x}=\left(\begin{array}{c}
X+a X^{2} / 2 \\
Y+b Y^{2} / 2 \\
Z(1+a X)^{-1}(1+b Y)^{-1}
\end{array}\right)
$$

as the solution deformation, for some parameters $a$ and $b$. We let the solution pressure be $p=2 c$, and specify displacement boundary conditions on the surface $X=0$, with traction boundary conditions on the remaining five surfaces. It is straightforward to determine the body force and surface tractions that are required to produce this deformation, and they are given for reference in the Appendix. 


\subsubsection{Model Problem 2: Mixed shears and discontinuous material properties}

As previously mentioned, a model of the breast can be comprised of various tissues - typically fat and fibroglandular, but skin and tumour are also possible - each with different material properties. Given a mesh created using the breast geometry, it is natural to specify a tissue type to each element. Motivated by this, we now suppose the body has discontinuous material properties, specifically $c$ in (1) satisfying $c=c_{1}$ for $X \leq 0.5$ and $c=c_{2}$ for $X>0.5$. We choose a problem with a known solution and continuous data, i.e. continuous body force and surface traction. We fix the left-hand edge $\left(\boldsymbol{u}^{*}=0\right.$ on $\left.X=0\right)$, and pick a solution which is a mixture of two shears, with the bottom surface having gradient $-\alpha$ for $X \leq 0.5$, and gradient $-\beta$ for $X>0.5$ :

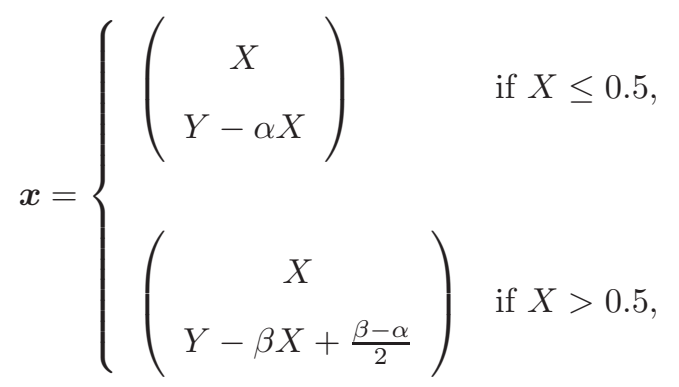

as shown in Figure 3(b). The deformation gradient and 1st Piola-Kirchhoff stress can then be computed to be

$$
\mathbf{F}=\left\{\begin{array}{l}
\left(\begin{array}{cc}
1 & 0 \\
0 & 1-\alpha
\end{array}\right) \quad \text { if } X \leq 0.5, \\
\left(\begin{array}{cc}
1 & 0 \\
0 & 1-\beta
\end{array}\right) \quad \text { if } X>0.5,
\end{array}\right.
$$


and

$$
\mathbf{S}=\left\{\begin{array}{cc}
\left(\begin{array}{cc}
2 c_{1}-p & -2 c_{1} \alpha \\
-p \alpha & 2 c_{1}-p
\end{array}\right) \quad \text { if } X \leq 0.5, \\
\left(\begin{array}{cc}
2 c_{2}-p & -2 c_{2} \beta \\
-p \beta & 2 c_{2}-p
\end{array}\right) \quad \text { if } X>0.5 .
\end{array}\right.
$$

We choose $p(\boldsymbol{X})=2 c_{1}$ for $X \leq 0.5$. The surface tractions on the top and bottom surface are then

$$
\boldsymbol{s}=\left(\begin{array}{c} 
\pm 2 c_{1} \alpha \\
0
\end{array}\right) \text { on } Y=0,1 ; X \leq 0.5
$$

(the positive choice for $Y=0$ and negative choice for $Y=1$ ). We need to choose $p$ in $X>0.5$, and $\beta$, so that the surface tractions are constant on $Y=0,1$ for all $X$; this requires $p=2 c_{2}$ in $X>0.5$ and $\beta=c_{1} \alpha / c_{2}$. Then $\mathbf{S}$ is constant in space (not just piecewise constant), so (3) is satisfied with zero body force, and the surface tractions given by (10) hold for all $X$. Finally, the surface traction on the right-hand edge is

$$
s=\left(\begin{array}{c}
0 \\
-2 c_{1} \alpha
\end{array}\right) \text { on } X=1 .
$$

Note that for this problem, $\boldsymbol{x}$ is continuous but has a discontinuous derivative, $p$ is discontinuous, but $\mathbf{S}$ and $s$ are continuous. 


\subsubsection{Model Problem 3: Steep changes in $x$}

The deformation of electrically-activated cardiac tissue is forced by a term which is added to the 2nd Piola-Kirchhoff stress:

$$
\mathbf{T}_{\text {cardiac }}=T_{a} \boldsymbol{f} \boldsymbol{f}^{\mathrm{T}}
$$

where $\boldsymbol{f}(\boldsymbol{X})$ is the unit direction of the cardiac fibre passing through $\boldsymbol{X}$, and $T_{a}$ is the active tension, which depends on physiological parameters such as intracellular calcium ion concentration, and becomes non-zero when the cell is excited. The deformation of the tissue can be modelled assuming quasi-steady elasticity [37] and therefore using (3), and, at any particular time, the solution deformation can have localised regions of compression, as explained in Section 1. This means that $\mathbf{T}_{\text {cardiac }}$ (and the body force that $\mathbf{T}_{\text {cardiac }}$ is equivalent to) have high spatial gradients. In the third model problem, we choose a deformation which changes steeply in one region. Let $\mu(X)=\frac{d}{2}\left(\tanh \left(k\left(X-\frac{1}{2}\right)\right)+1\right)$, for some large $k$ and $d>0$, which satisfies $\mu \approx 0$ for $X<0.5$ and $\mu \approx d$ for $X>0.5$, with a sharp increase at $X \approx 0.5$. Let us choose as the solution

$$
\boldsymbol{x}=\left(\begin{array}{c}
X-\mu \\
\frac{Y}{1-\mu^{\prime}}
\end{array}\right), \quad p=2 c
$$

as shown in Figure 3(c), which, for suitably large $k$, represents very little displacement for $X<0.5$, a sharp compression in the $X$-direction and elongation in the $Y$-direction around $X \approx 0.5$, and only a translation for $X>0.5$. We choose corresponding displacement boundary conditions at $X=0: \boldsymbol{u}^{*}=$ $\left(-\mu(0), \quad Y-Y /\left(1-\mu^{\prime}(0)\right)\right)^{\mathrm{T}}$, which is effectively $\boldsymbol{u}^{*}=0$ on $X=0$ if $k$ is large. Defining $\lambda=1-\mu^{\prime}$, the deformation gradient and 1st Piola-Kirchhoff 
stress are then

$$
\mathbf{F}=\left(\begin{array}{cc}
\lambda & 0 \\
Y \mu^{\prime \prime} \lambda^{-2} & \lambda^{-1}
\end{array}\right), \quad \mathbf{S}=2 c\left(\begin{array}{cc}
\lambda-\lambda^{-1} & Y \mu^{\prime \prime} \lambda^{-2} \\
Y \mu^{\prime \prime} \lambda^{-2} & \lambda^{-1}-\lambda
\end{array}\right)
$$

and again $\mathbf{F}$ has unit determinant as required. The required body force and surface tractions are then, using (3) and (6):

$$
\begin{aligned}
& \boldsymbol{b}=\frac{2 c}{\rho_{0}}\left(\begin{array}{c}
\mu^{\prime \prime} \\
-Y \mu^{\prime \prime \prime} \lambda^{-2}-2 Y\left(\mu^{\prime \prime}\right)^{2} \lambda^{-3}
\end{array}\right), \\
& s= \begin{cases}2 c\left(\begin{array}{c}
0 \\
\lambda-\lambda^{-1}
\end{array}\right) & \text { if } Y=0, \\
2 c\left(\begin{array}{c}
\mu^{\prime \prime} \lambda^{-2} \\
\lambda^{-1}-\lambda
\end{array}\right) & \text { if } Y=1, \\
2 c\left(\begin{array}{c}
\lambda-\lambda^{-1} \\
Y \mu^{\prime \prime}(1) \lambda(1)^{-2}
\end{array}\right) & \text { if } X=1 .\end{cases}
\end{aligned}
$$

\subsubsection{Model Problem 4: Discontinuous applied tractions}

Our fourth model problem is motivated by the modelling of mammography, where the breast is very heavily compressed by two plates with normals in the direction of the X-ray beam, in order to spread out the tissue. The resultant deformation is very large, hindering easy comparison with MR images (for example), and software which could predict the deformation would be an important aid in diagnosis. Since the part of the skin that will be in contact with the compression plates is not known in advance, the problem cannot be stated simply as an elasticity problem with displacement or traction boundary conditions. 
Instead, it has to be stated as a constrained elasticity problem. Such problems are known as contact problems, and can be solved using an energy minimisation with inequality constraints procedure, such as the Augmented Lagrangian method [38], used to model breast-mammographic deformation in [35]. Here, we consider a simplified situation where we do apply a known surface traction. We take this traction to be discontinuous, since the deformation of the breast surface in mammography is discontinuous at the edge of the compression plate, and $\mathbf{S}^{\mathrm{T}} \boldsymbol{N}$ must also be discontinuous there. Unfortunately it is extremely difficult to construct a deformation which is incompressible and has a discontinuity in the derivative at one (non-corner) point on the boundary, and therefore we cannot start from a known solution in this model problem. Instead, we choose to fix the bottom surface $\left(\boldsymbol{u}^{*}=0\right.$ on $\left.Y=0\right)$, apply zero body force $(\boldsymbol{b}=0)$, and apply the surface traction

$$
s= \begin{cases}\left(\begin{array}{c}
0 \\
-3 c
\end{array}\right) & \text { if } 0.25 \leq X \leq 0.75 \text { and } Y=1 \\
\left(\begin{array}{c}
0 \\
0
\end{array}\right) & \text { otherwise }\end{cases}
$$

The solution of this problem is displayed in Figure 3(d). (Note that here, unlike Figures 3(a)-(c), a numerically-computed solution is plotted).

\section{Results}

For each of the first three model problems, in which the analytic solution is known, we choose a point $\boldsymbol{X}^{*}$ and measure the error

$$
e=\left\|\boldsymbol{x}^{\text {computed }}\left(\boldsymbol{X}^{*}\right)-\boldsymbol{x}^{\text {exact }}\left(\boldsymbol{X}^{*}\right)\right\|_{2},
$$


for each of the different numerical schemes. For schemes requiring quadrilateral meshes, we discretise the unit square into $N$ by $N$ uniform square elements (or uniform cubes in $3 \mathrm{D})$. In this case the spatial stepsize is $h=1 / N$. For the scheme requiring a triangular mesh, we discretise the unit square into $N$ by $N$ squares and then subdivide each square into 2 triangles (or 6 tetrahedra in 3D). Therefore, we define the spatial stepsize in this case to also be $h=1 / N$. We then plot the error against $h$.

\subsection{Model Problem 1a}

For the first model problem, we choose $a=0.2$ and $\boldsymbol{X}^{*}=(1,1)$. The error against $h$ when solving this problem using each of the different schemes is plotted in Figure 4.

In this model problem the solution displacement and pressures are smooth without sharp changes, and therefore we would expect smooth convergence as $h$ decreases, at the rate determined by the degree of basis function used. This behaviour is indeed exhibited, with $\log (e)$ varying linearly with $\log (h)$ for each scheme, and the gradient approximately equal to 2 for the linear scheme, 3 for the quadratic schemes, and very near 4 for the cubic-Hermite scheme, i.e. convergence is close to $\mathcal{O}\left(h^{2}\right), \mathcal{O}\left(h^{3}\right)$ and $\mathcal{O}\left(h^{4}\right)$ respectively, which is the behaviour of these schemes predicted for linear problems by error analysis. We see also that the choice of element when using a quadratic scheme has a big impactthe two quadratic schemes converge at the same rate but the quad-quad scheme performs better than the quad-tri method, being an order of magnitude more accurate at comparable stepsizes. The cubic-Hermite scheme, which has the same number of degrees of freedom for a given stepsize as the quadratic scheme, performs even better, providing extremely accurate solutions even when the number of nodes is small. 


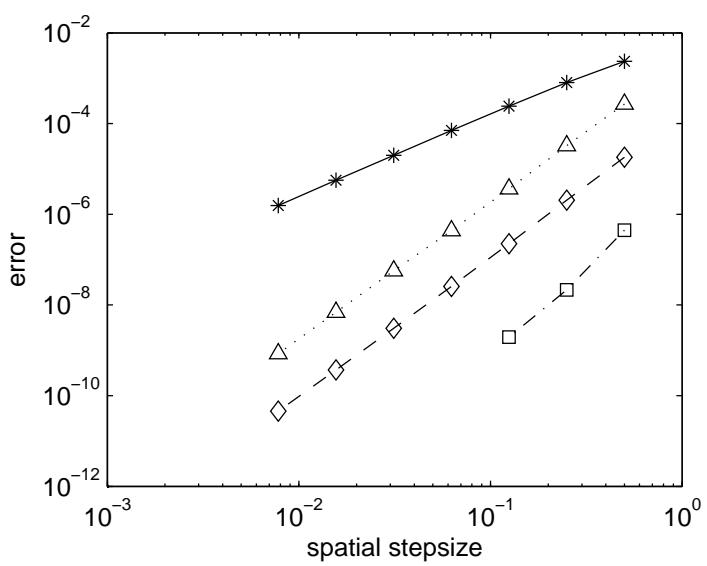

Figure 4: Results with Model Problem 1a: error plotted against spatial stepsize, using the: linear method (solid lines with stars); quad-tri (dotted lines with triangles); quad-quad method (dashed lines with diamonds); and cubic-Hermite method (dot-dashed lines with squares).

This first model problem verifies that the implementations of the four different numerical methods are correct, and illustrates the 'best-case' convergence behaviour, and will thus enable us to quantitatively compare error magnitudes and rates of convergence in the other, biologically-motivated, model problems.

\subsection{Model Problem 1b}

For this model problem, we choose $a=b=0.1$ and $\boldsymbol{X}^{*}=(1,1,1)$. The error against $h$ using each of the different schemes is plotted in Figure 5. We see that the linear, quad-tri and quad-quad schemes $^{2}$ perform similarly to in twodimensions, with similar rates of convergence to $2 \mathrm{D}$, and with the magnitude of the error decreasing significantly from the linear scheme to the quad-tri scheme, and from quad-tri to quad-quad. In 3D, the error using the quad-quad scheme is actually two order of magnitudes smaller than the quad-tri error using the

\footnotetext{
${ }^{2}$ The latter two methods should perhaps now be referred to as the quad-tet and quad-hex schemes, as in 3D they are quadratic bases on tetrahedra and hexahedra, but for simplicity we retain the $2 \mathrm{D}$ names.
} 
same spatial stepsize, compared to one order of magnitude in 2D. However, the cubic-Hermite scheme performs relatively badly on this problem. Here, the convergence rate is approximately the same as the lower order quadratic schemes, which may be explained by the fact that the cubic-Hermite scheme, similarly to the quadratic methods, only uses a linear approximation for pressure. However, it is not clear why the magnitude of the error is significantly greater than with the other methods, and requires further investigation, which we leave for future work. Although this poor performance is surprising, cubic hermite basis functions have not been employed as often as conventional finite elements, and have received correspondingly less analytical attention.

Unfortunately, these results therefore do not allow us to state that the following conclusions regarding the four schemes on Model Problems 2-4 can be assumed to hold in 3D as well as 2D, and further investigation will need to take place. However, these 3D results do at least suggest that the relative behaviour of the linear, quad-tri and quad-quad schemes is similar in 3D to 2D, and that, if the cubic-Hermite method performs poorly on a $2 \mathrm{D}$ model problem, it will perform poorly on a $3 \mathrm{D}$ problem.

We also use this $3 \mathrm{D}$ model problem to consider the computational cost of each method. Note that since different programming languages, programming styles, libraries and machines were used when implementing these models and running the simulations, it would be uninformative to simply compare computation times. Now, in each Newton iteration, two tasks take up the majority of the computational work: assembling a Jacobian matrix, and solving a linear system for the update vector (see [35] for more details). We only look at the work done in assembling the Jacobian, which generally dominates the work done in solving the linear systems for the simulations in this paper. This involves four nested loops: a loop over all elements; a loop over quadrature points; and two 


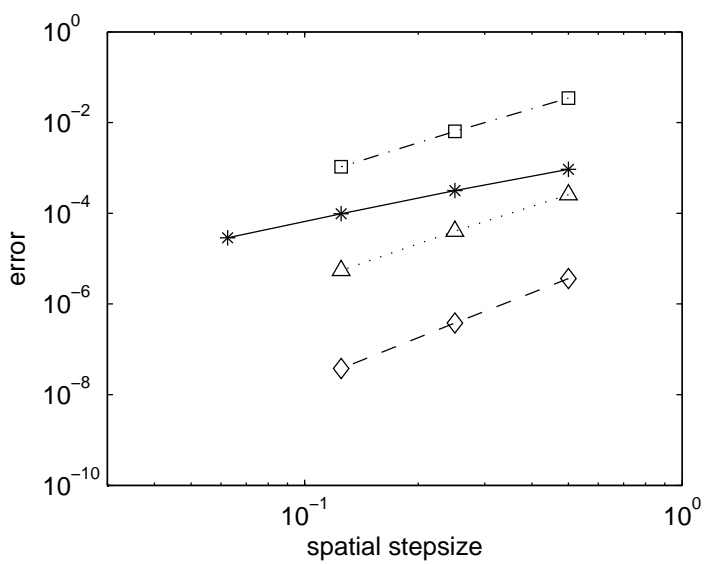

Figure 5: Results with Model Problem 1b (3D): error plotted against spatial stepsize, using the: linear method (solid lines with stars); quad-tri (dotted lines with triangles); quad-quad method (dashed lines with diamonds); and cubicHermite method (dot-dashed lines with squares).

loops over the unknowns per element (in order to fill in a 2D array of size $N_{u}$ by $N_{u}$, where $N_{u}$ is the number of unknowns per element). The work done within these loops is approximately equal between the four methods. Therefore, we can approximate the dominating component of the computational work for each method by $W_{c}=k M K N_{u}^{2}$, where $M$ is the total number of elements, $K$ the total number of quadrature points per element, and $k$ is a constant. To determine suitable values of $K$, we have calculated the error using this model problem with $h=1 / 4$, using a selection of values in the number of quadrature points in each direction, the results of which are given in Table 2. The table clearly shows that 2 quadrature points in each direction $(\Rightarrow K=8)$ is the most suitable trade-off between speed and accuracy for the linear method, whereas 3 $(\Rightarrow K=27)$ is the best choice for the other methods (despite cubic-Hermite basis functions being higher-order than quadratic bases). Also, $N_{u}=3 \times 8+1=25$ for the linear method (in 3D), $N_{u}=34$ for the quad-tri method, $N_{u}=68$ for the quad-quad method, and $N_{u}=200$ for the cubic-Hermite method. Putting 


\begin{tabular}{c|cccc} 
Method & \multicolumn{4}{|c}{ Number of quadrature points in each direction } \\
& 1 & 2 & 3 & 4 \\
\hline Linear & fail & $3.1618 \times 10^{-4}$ & $3.1612 \times 10^{-4}$ & \\
Quad-tri & fail & $2.3370 \times 10^{-3}$ & $4.0321 \times 10^{-5}$ & $4.0320 \times 10^{-5}$ \\
Quad-quad & fail & fail & $3.7986 \times 10^{-7}$ & $3.7968 \times 10^{-7}$ \\
Cubic-Hermite & & fail & $6.3710 \times 10^{-3}$ & $6.3706 \times 10^{-3}$
\end{tabular}

Table 2: Errors in Model Problem 1b for each of the four numerical schemes using various numbers of quadrature points (in each direction) for the numerical integration. Here 'fail' indicates failure of the method to converge.

all this together, and defining $k_{2}=200 k$, we obtain

$$
W_{c}= \begin{cases}k_{2} M & \text { for the linear scheme } \\ 4.59 k_{2} M & \text { for the quad-tri scheme } \\ 9.18 k_{2} M & \text { for the quad-quad scheme } \\ 27 k_{2} M & \text { for the cubic-Hermite scheme. }\end{cases}
$$

This information can be used together with a convergence plot to determine the best choice of method for a particular problem. For example, the quadquad method is certainly the best choice of method for Model Problem 1b. For example, linearly extrapolating the linear results suggest that a spatial stepsize approximately of $h=0.01$ (corresponding to $M=10^{6}$ ) would be required for the linear method to give as accurate results as the quad-quad method with $h=0.5$ (corresponding to $M=8$ hexahedral elements), which translates to $W_{c}$ being approximately $10^{5}$ times greater for the linear method than the quad-quad method. Similarly, the quad-tri method can be shown to be far less efficient than the quad-quad method on this problem.

\subsection{Model Problem 2}

For Model Problem 2, we choose $\alpha=0.1, c_{1} / c_{2}=5$ (which implies that $\beta=0.5$ ), and $\boldsymbol{X}^{*}=(1,1)$. The error in this problem against $h$ is given in Figures $6(\mathrm{a})$ 
and (b).

In this problem we had discontinuous material parameters but a smooth body force and surface traction, which resulted in a solution with a discontinuous derivative of displacement, and discontinuous pressure. For the schemes compared, only the linear scheme allows a discontinuous solution pressure, since pressure is interpolated using element-wise rather than node-wise values. In fact, the exact solution is within the space spanned by the basis functions in the linear case, and therefore the finite element solution should be the exact solution (to within machine precision), and it can be seen that the linear scheme does indeed find the exact solution for all stepsizes $h$. (This, of course, requires that $N$ be even, so that no element crosses the material interface). By contrast, the quad-tri, quad-quad and cubic-Hermite methods all perform extremely poorly compared to the linear method, exhibiting large errors and low rates of convergence. Here, the gradient of $\log (e)$ against $\log (h)$ is approximately 1 for each of the quadratic and cubic-Hermite schemes, so convergence is only $\mathcal{O}(h)$. In fact, each of these three schemes perform almost identically on this problem. We see that the benefits of using a higher-order method have been lost due to the scheme not being able to accurately approximate $p$. Note, however, that while the cubic-Hermite scheme might have been expected to perform worse than the quadratic schemes on this problem (since the cubic-Hermite scheme also enforces continuity in the derivative of the displacement), it actually provides marginally more accurate solutions than the quadratic schemes.

For illustrative purposes, the deformed nodal locations when the quad-tri method is used with $h=1 / 16$ are plotted in Figure 7, which clearly displays significant error at the material interface, despite a reasonably fine mesh. 


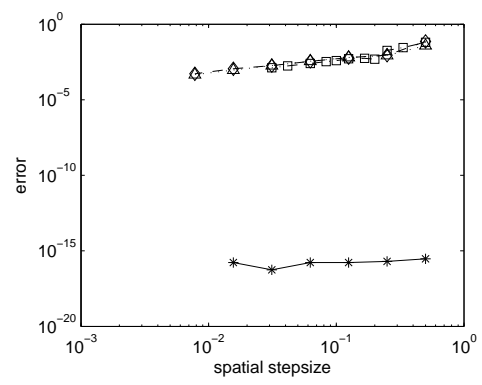

(a)

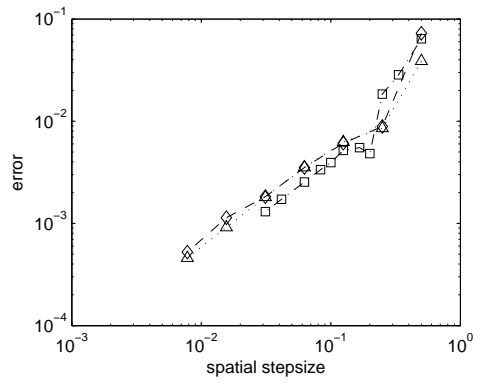

(b)

Figure 6: Results with Model Problem 2, error plotted against spatial stepsize. The two figures plot the same results except the linear method is not plotted in (b). Methods plotted are: the linear method (solid lines with stars); quad-tri (dotted lines with triangles); quad-quad method (dashed lines with diamonds); and cubic-Hermite method (dot-dashed lines with squares).

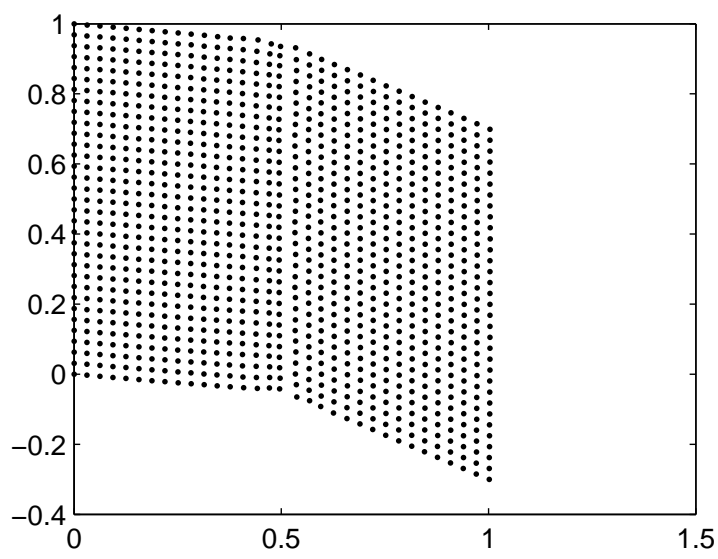

Figure 7: The location of the deformed nodes when the quad-tri method is used on Model Problem 2 with $h=1 / 16$. (Note that internal nodes are shown as well as vertices, which is why there are $33^{2}$ nodes rather than $17^{2}$ ). 


\subsection{Model Problem 3}

For this model problem, we choose $d=0.05, k=10$, and $\boldsymbol{X}^{*}=(0.5,1)$ (i.e. the top of the deforming area in Figure $3(\mathrm{c}))$. The error against $h$, and against the number of degrees of freedom in the scheme, are plotted in Figures 8(a) and (b) respectively.

Since the data in this problem - the material parameters, body force and surface tractions - are infinitely differentiable we might expect the convergence properties of the four schemes to be similar to that displayed for Model Problem 1a for small enough stepsizes (assuming exact arithmetic). However, for the range of stepsizes chosen (which are 'natural' choices of this size of domain), we see that the clear, well-defined behaviour of Model Problem 1a is not exhibited. The gradient of the linear scheme does settle down to near 2 for the smaller stepsizes (i.e. $\mathcal{O}(h)$ convergence), as in Model Problem 1a. By contrast, the gradient of the quad-tri method, using the last four grid-points, is actually 3.95, i.e. near $\mathcal{O}\left(h^{4}\right)$ convergence, strangely much faster convergence than would be expected. However, the quad-quad method appears to undergo numerical locking, with the final two points plotted showing barely-decreased error, despite the stepsize halving and quadrupling. It is not clear why this is the case. (Note that it has been verified that the Newton solver has fully converged to a solution which satisfies the nonlinear system to high very accuracy in this (and all other) cases). The cubic-Hermite method follows a similar path to the quad-tri method.

Overall, we see that there is not a huge difference between the four methods for this model problem, and certainly not the clear pronounced difference observed in Model Problem 1a. Figure 8(b) suggests that the choice of method is relatively unimportant when there is sharply-varying data that is resolved using the range of stepsizes we have chosen: for a given number of degrees of freedom, 


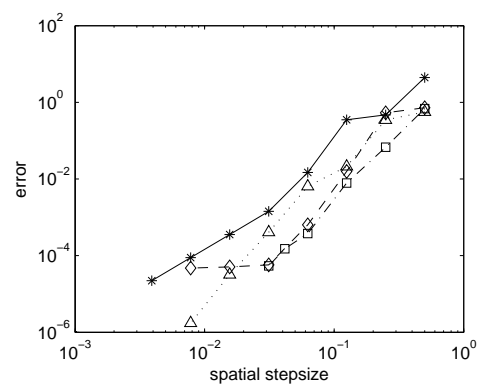

(a)

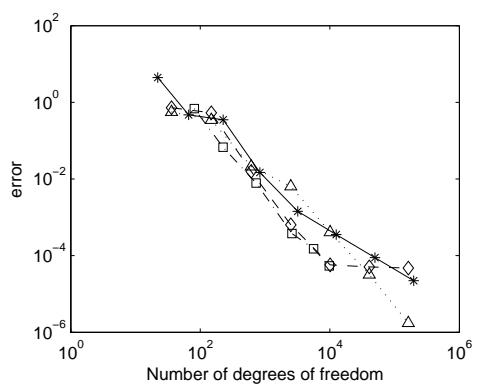

(b)

Figure 8: Results with Model Problem 3, error plotted against (a) spatial stepsize and (b) number of degrees of freedom. Methods plotted are: the linear method (solid lines with stars); quad-tri (dotted lines with triangles); quad-quad method (dashed lines with diamonds); and cubic-Hermite method (dot-dashed lines with squares).

the difference in the error between the four methods varies only by about one order of magnitude.

The results for this model problem indicate that the theoretical asymptotic convergence rates of finite element schemes should not be naïvely expected when modelling cardiac deformation. We leave for future work finite element convergence analysis of contracting cardiac tissue on realistic domains and/or with experimentally-derived parameters values.

\subsection{Model Problem 4}

For the final model problem, we do not have an exact error with which to quantify numerical behaviour and must choose an alternative approach. One quantity which can be measured, however, is the stress in the normal direction 


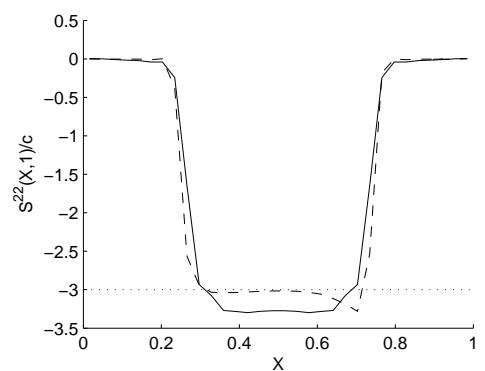

(a)

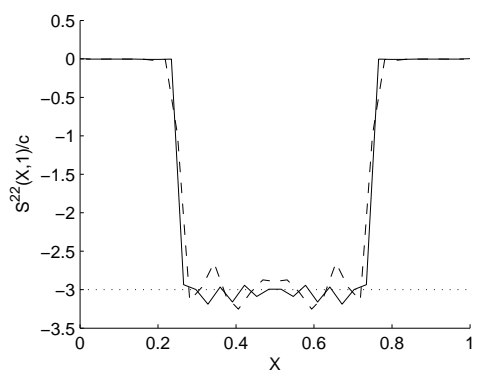

(b)

Figure 9: Results with Model Problem 4, the normal stress (scaled by $c$ ) on the top surface of the body. (a) The linear method (solid line) and quad-tri method (dashed line). (b) The quad-quad method (solid line) and cubic-Hermite method (dashed line). The dotted lines are just the lines $y=-3$.

on the top surface, $\boldsymbol{N}^{\mathrm{T}} \mathbf{S}^{\mathrm{T}} \boldsymbol{N}=S^{22}$. Using (6) and (11), this should satisfy

$$
S^{22}(X, 1)= \begin{cases}-3 c & \text { if } 0.25 \leq X \leq 0.75 \text { and } Y=1, \\ 0 & \text { otherwise. }\end{cases}
$$

Figure 9 plots $S^{22} / c$ on the top surface for each of the schemes, using $h=1 / 32$. Note that for the linear and quadratic schemes, the derivative of the numerical solution displacement is discontinuous at the nodes, and therefore $\mathbf{F}$ (and thus S) cannot be evaluated there. Instead, $S^{22}$ is evaluated at the mid-points of each top-surface boundary element. The cubic-Hermite method, however, provides the nodal values of the first derivatives of the displacement, so for this method the nodal values of $S^{22}$ are plotted.

The figure shows that solutions obtained using the different schemes are qualitatively different. For $0.25 \leq X \leq 0.75$, the stresses oscillate around the true value for the quad-quad and cubic-Hermite methods, but they do not for the linear and quad-tri methods. In most of this region, the error for the quad-tri method is significantly smaller than for the linear method. The cubic- 
Hermite scheme is seen to perform relatively poorly on this problem, especially in comparison with the quadratic methods (which have the same number of degrees of freedom as the cubic-Hermite method), with large oscillations around the correct stress. This can be attributed to the fact that the cubic-Hermite method enforces continuity of the derivative of $\boldsymbol{x}$, whereas the true solution has a discontinuous derivative of $\boldsymbol{x}$ on the top surface.

In Figure 10(a), we also plot the value of $y(0.5,1)$ against the stepsize $h$ for the four schemes, in order to qualitatively compare the rates of convergence. Although all methods converge to approximately the same value, we see that the cubic-Hermite method provides the least accurate solution for some of the larger stepsizes (in complete contrast to the results for Model Problem 1a). For $h=1 / 16$, the errors for the linear and cubic-Hermite schemes are approximately equal, and greater than the errors for the two quadratic schemes, also approximately equal. Finally, in Figure 10(b), we quantify the errors in $S^{22}$. Let the average error in $S^{22} / c$ be defined as

$$
e_{S}=\frac{1}{M c} \sum_{X}\left|S^{22}(X)-S_{\text {exact }}^{22}(X)\right|
$$

where $S_{\text {exact }}^{22}$ is given by the right-hand side of (12) and $M$ is the number of evaluation points. Note that $e_{S}$ is dominated by errors in $S^{22}$ near the discontinuities $(X=0.25$ and $X=0.75)$, and due to the difference in how the evaluation points are chosen for the cubic-Hermite method compared to the other methods (as described at the beginning of this section), it would not be a fair comparison to compare this error for the cubic-Hermite scheme against the others. We therefore only plot $e_{S}$ for the linear and quadratic schemes. Figure 10(b) shows that the quad-quad method performs significantly better than the quad-tri method, which performs significantly better than the linear method, similarly to the first model problem. 


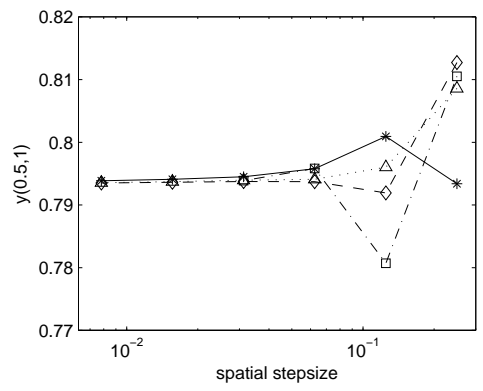

(a)

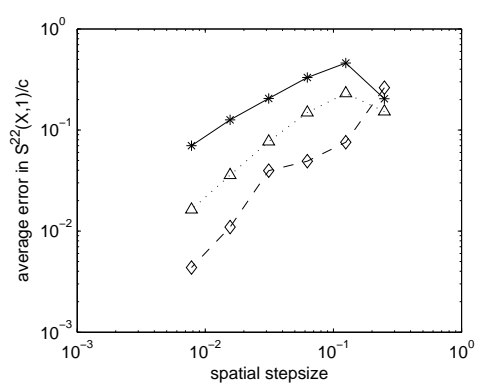

(b)

Figure 10: Results with Model Problem 4: (a) $y(0.5,1)$ plotted against spatial stepsize and (b) the average error in $S^{22} / c$ against stepsize. Methods plotted are: the linear method (solid lines with stars); quad-tri (dotted lines with triangles); quad-quad method (dashed lines with diamonds); and cubic-Hermite method (dot-dashed lines with squares) (not plotted in (b)).

\section{Discussion}

We have performed a study of the numerical behaviour of four commonly-used finite element schemes on four model problems, three of which were constructed to replicate deformations which occur in soft-tissue modelling of the heart and breast. Overall, we have shown that in soft-tissue modelling, it is important to take the expected regularity of the solution into account when choosing a numerical scheme.

In the first model problem, the best-case convergence behaviour of the schemes was illustrated, as the material parameters, body force and surface tractions were all smooth, and the convergence rates were close to that predicted for linear problems - $\mathcal{O}\left(h^{2}\right)$ for the linear method, $\mathcal{O}\left(h^{3}\right)$ for the quadratic methods, and $\mathcal{O}\left(h^{4}\right)$ for the cubic-Hermite method. The quad-quad method was also found to be an order of magnitude more accurate than the quad-tri method. A 3D version of this model problem was also studied, for which the linear, quadtri and quad-quad methods performed similarly to 2D, but the cubic-Hermite method performed relatively poorly. We have also studied the computational 
cost of each method, determining that the ratio of computational work per element required to assemble the Jacobian matrix (which dominates the total computational cost) is approximately 1:5:10:30 (linear to quad-tri to quad-quad to cubic-Hermite).

We have shown that if material parameters becomes discontinuous, as is the case when modelling breast deformation, where different internal tissues (for example fat and fibroglandular tissue) have different material properties, the solution pressure is discontinuous (although the stresses may not be), and the quadratic and cubic-Hermite methods all perform poorly and only display $\mathcal{O}(h)$ convergence, since they enforce continuity of the pressure. However, despite the cubic-Hermite method also incorrectly enforcing continuity of the derivative of displacement, it does not perform significantly worse than the quadratic methods. For the particular model problem set up, the linear method, which allowed a discontinuous pressure, was able to find the true solution to within machine precision.

An alternative FE method that may be appropriate under these conditions is the discontinuous Galerkin FE method (DGFEM). This FE method, whose development is much less mature than the more common continuous Galerkin FE method, allows discontinuities in the solution across element boundaries. Recent work that has applied DGFEM to nearly incompressible linear elasticity [39] and nearly incompressible nonlinear elasticity [40] has demonstrated no evidence of locking for a linear approximation to the displacement on each element. As such, future work should investigate the applicability of DGFEM to fully incompressible nonlinear elasticity. Another FE method which may be effective in soft tissue models with material interfaces and discontinuous pressures, is the Extended FE method (XFEM), which has been developed to handle solution discontinuities without the need for local mesh refinement [41]. 
A related problem is Model Problem 4, where a discontinuous surface traction was applied - motivated by modelling the compression of the breast during mammography — for which the solution has a discontinuous first derivative of displacement but continuous pressure. In this problem the regularity of the finite element solutions in the linear and quadratic schemes matched the regularity of the solution, but the cubic-Hermite method incorrectly enforces continuity of the derivative of position. The cubic-Hermite method still found reasonable approximations to the solution, but it was not as accurate as the quadratic schemes.

Finally, we also considered a model problem in which the solution and body force, although smooth, varied sharply across the domain, motivated by the deformation of cardiac tissue. The clear, distinct, best-case convergence behaviour of the schemes on Model Problem 1a was not observed; instead the four schemes all performed loosely the same, especially when the number of degrees of freedom was taken into account.

\section{Appendix}

For the three-dimensional model problem, Model Problem 1b, we let $\Omega_{0}$ be the unit cube, and define

$$
\boldsymbol{x}=\left(\begin{array}{c}
X+a X^{2} / 2 \\
Y+b Y^{2} / 2 \\
Z(1+a X)^{-1}(1+b Y)^{-1}
\end{array}\right)
$$


as the deformation in 3D, for some parameters $a$ and $b$. Defining $\lambda_{1}=1+a X$ and $\lambda_{2}=1+b Y$, the deformation gradient is given by

$$
\mathbf{F}=\left(\begin{array}{ccc}
\lambda_{1} & 0 & 0 \\
0 & \lambda_{2} & 0 \\
-a Z \lambda_{1}^{-2} \lambda_{2}^{-1} & -b Z \lambda_{1}^{-1} \lambda_{2}^{-2} & \lambda_{1}^{-1} \lambda_{2}^{-1}
\end{array}\right)
$$

which has inverse

$$
\mathbf{F}^{-1}=\left(\begin{array}{ccc}
\lambda_{1}^{-1} & 0 & 0 \\
0 & \lambda_{2}^{-1} & 0 \\
a Z \lambda_{1}^{-2} & b Z \lambda_{2}^{-2} & \lambda_{1} \lambda_{2}
\end{array}\right)
$$

Using the same Neo-Hookeon strain energy (1) (except with (tr $\mathbf{C}-3$ ) instead of ( $\operatorname{tr} \mathbf{C}-2)$, although this has no effect on the stresses), (2) still holds, and so, letting $p=2 c$ again, we have

$$
\mathbf{S}=2 c\left(\begin{array}{ccc}
\lambda_{1}-\lambda_{1}^{-1} & 0 & -a Z \lambda_{1}^{-2} \lambda_{2}^{-1} \\
0 & \lambda_{2}-\lambda_{2}^{-1} & -b Z \lambda_{1}^{-1} \lambda_{2}^{-2} \\
-a Z \lambda_{1}^{-2} & -b Z \lambda_{2}^{-2} & \lambda_{1}^{-1} \lambda_{2}^{-1}-\lambda_{1} \lambda_{2}
\end{array}\right)
$$

Assuming we apply displacement boundary conditions on the surface $X=0$ and traction boundary conditions on the remaining five surfaces, the problem to be solved therefore involves specifying body force

$$
\boldsymbol{b}=-\frac{2 c}{\rho_{0}}\left(\begin{array}{c}
a \\
b \\
2 Z \lambda_{1}^{-1} \lambda_{2}^{-1}\left(a^{2} \lambda_{1}^{-2}+b^{2} \lambda_{2}^{-2}\right)
\end{array}\right)
$$


and applying boundary conditions

$$
\begin{array}{ll}
\boldsymbol{x}=\left(\begin{array}{ccc}
0, & Y+b Y^{2} / 2, & Z \lambda_{2}^{-1}
\end{array}\right)^{\mathrm{T}} & \text { on } X=0, \\
s=S^{\mathrm{T}} \mathbf{N}=2 c\left(\begin{array}{lll}
\lambda_{1}-\lambda_{1}^{-1}, & 0, & -a Z \lambda_{1}^{-2} \lambda_{2}^{-1}
\end{array}\right)^{\mathrm{T}} & \text { on } X=1, \\
s=S^{\mathrm{T}} \mathbf{N}=2 c\left(\begin{array}{lll}
0, & 0, & b Z \lambda_{1}^{-1}
\end{array}\right)^{\mathrm{T}} & \text { on } Y=0, \\
s=S^{\mathrm{T}} \mathbf{N}=2 c\left(\begin{array}{lll}
0, & \lambda_{2}-\lambda_{2}^{-1}, & -b Z \lambda_{1}^{-1} \lambda_{2}^{-2}
\end{array}\right)^{\mathrm{T}} & \text { on } Y=1, \\
s=S^{\mathrm{T}} \mathbf{N}=2 c\left(\begin{array}{lll}
0, & \lambda_{1} \lambda_{2}-\lambda_{1}^{-1} \lambda_{2}^{-1}
\end{array}\right)^{\mathrm{T}} & \text { on } Z=0, \\
s=S^{\mathrm{T}} \mathbf{N}=2 c\left(\begin{array}{lll}
-a \lambda_{1}^{-2}, & -b \lambda_{2}^{-2}, & \lambda_{1}^{-1} \lambda_{2}^{-1}-\lambda_{1} \lambda_{2}
\end{array}\right)^{\mathrm{T}} & \text { on } Z=1 .
\end{array}
$$

\section{Acknowledgments}

PP is supported through the EPSRC-funded OXMOS project New frontiers in the mathematics of solids, (grant reference EP/D048400/1). JPW is partially funded by the EPSRC under grant EP/D503035/1. This publication is based on work supported by Award No. KUK-C1-013-04, made by King Abdullah University of Science and Technology (KAUST).

\section{References}

[1] Dehghani, H., Doyley, M., Pogue, B., Jiang, S., Geng, J., and Paulsen, K. Breast deformation modelling for image reconstruction in near infrared optical tomography. Phys. Med. Biol., 2004, 49, 1131-1145. 
[2] Washington, C., and Miga, M. Modality independent elastography (MIE): A new approach to elasticity imagining. IEEE Transactions on Medical Imaging, 2004, 23(9), 1117-1128.

[3] Papademetris, X., Sinusas, A., Dione, D., and Duncan, J. Estimation of 3D left ventricular deformation from echocardiography, Medical Image Analysis, 2001, 5(1), 17-28.

[4] Fung, Y. Biomechanics: Mechanical Properties of Living Tissues, 1993, Springer-Verlag, New York.

[5] Humphrey, J. Review Paper: Continuum biomechanics of soft biological tissues, Proceedings of the Royal Society A: Mathematical, Physical and Engineering Sciences, 2003, 459(2029), 3-46.

[6] Pathmanathan, P., Gavaghan, D., Whiteley, J., Chapman, S. J., and Brady, J. M. Predicting tumor location by modeling the deformation of the breast, IEEE Transactions on Biomedical Engineering, 2008, 55(10), $2471-2480$.

[7] Samani, A., Bishop, J., Yaffe, M., and Plewes, D. Biomechanical 3-d finite element modeling of the human breast using MRI data, IEEE Transactions on Medical Imaging, 2001, 20(4), 271-279.

[8] Azar, F., Metaxas, D., and Schnall, M. A deformable finite element model of the breast for predicting mechanical deformations under external perturbations, Academic Radiology, 2001, 8(10), 965-975.

[9] Kerckhoffs, R., Healy, S., Usyk, T., and McCulloch, A. Computational methods for cardiac electromechanics, Proceedings of the IEEE, 2006, 94(4), 769-783. 
[10] Nash, M. and Panfilov, A. Electromechanical model of excitable tissue to study reentrant cardiac arrhythmias, Progress in Biophysics and Molecular Biology, 2004, 85, 501-522.

[11] Nickerson, D., Smith, N. and Hunter, P. New developments in a strongly coupled cardiac electromechanical model. Europace, 2005, 7, S118$\mathrm{S} 127$.

[12] Oomens, C., Maenhout, M., van Oijen, C., Drost, M. and Baaijens, F. Finite element modelling of contracting skeletal muscle. Philosophical Transactions of the Royal Society of London B, 2003, 358(1437), $1453-1460$.

[13] Gardiner, J. and Weiss, J. Subject-specific finite element analysis of the human medial collateral ligament during valgus knee loading. Journal of Orthopaedic Research, 2003, 21(6), 1098-1106.

[14] Goreham-Vossb, C., McKinleya, T. and Brown, T. A finite element exploration of cartilage stress near an articular incongruity during unstable motion. J. Biomechanics, 2007, 40(15), 3438-3447.

[15] Picinbono, G., Delingette, H., and Ayache, N. Nonlinear and anisotropic elastic soft tissue models for medical simulation, IEEE International Conference on Robotics and Automation, 2001, 2, 1370-1375.

[16] Fung, Y. Elasticity of soft tissues in simple elongation, American Journal of Physiology, 1967, 213(6), 1532-1544.

[17] Zhou, J. and Fung, Y. The degree of nonlinearity and anisotropy of blood vessel elasticity, Proceedings of the National Academy of Sciences, 1997, 94(26), 14255-14260. 
[18] Ambrosi, D. and Mollica, F. The role of stress in the growth of a multicell spheroid, Journal of Mathematical Biology, 2004, 48(5), 477-499.

[19] Reddy, N. An Introduction to the Finite Element Method, 1993, McGrawHill, New York.

[20] Usyk, T. and McCulloch, A. Computational methods for soft tissue biomechanics, Courses and lectures-International Centre of Mechanical Sciences, 2003, 273-342, Springer-Verlag.

[21] Brezzi, F. and Fortin, M. Mixed and Hybrid Finite Element Methods, 1991, Springer-Verlag, New York.

[22] Chapelle, D. and Bathe, K. The inf-sup test, Computers and structures, $1993,47,537-537$.

[23] Benzley, S., Perry, E., Merkley, K., Clark, B. and Sjaardema, G. A comparison of all-hexahedral and all-tetrahedral finite element meshes for elastic and elasto-plastic analysis. In Proceedings, 4th International Meshing Roundtable, 1995, 179-191.

[24] Ramos, A. and Simões, J. Tetrahedral versus hexahedral finite elements in numerical modeling of the intact femur. Med Eng Phys, 2006, 28, 916924.

[25] Elman, H., Silvester, D. and Wathen, A. Finite elements and fast iterative solvers: with applications in incompressible fluid dynamics, 2005, Oxford University Press, Oxford.

[26] Whiteley, J. The solution of inverse non-linear elasticity problems that arise when locating breast tumours, Computational and Mathematical Methods in Medicine, 2005,6(3), 143-149. 
[27] Whiteley, J., Gavaghan, D., Chapman, S. J. and Brady, J. M. Nonlinear modelling of breast tissue, Math Med Biol, 2007, 24(3), 327-345.

[28] Donzelli, P. and Spilker, R. A contact finite element formulation for biological soft hydrated tissues, Computer Methods in Applied Mechanics and Engineering, 1998, 153(1-2), 63-79.

[29] Driessen, N., Mol, A., Bouten, C. and Baaijens, F. Modeling the mechanics of tissue-engineered human heart valve leaflets, Journal of Biomechanics, 2007, 40(2), 325-334.

[30] Bovendeerd, P., Arts, T., Delhaas, T., Huyghe, J., van Campen, D. and Reneman, R. Regional wall mechanics in the ischemic left ventricle: numerical modeling and dog experiments, American Journal of Physiology- Heart and Circulatory Physiology, 1996, 270(1), 398-410.

[31] Nash, M. Mechanics and Material Properties of the Heart using an Anatomically Accurate Mathematical Model, $\mathrm{PhD}$ thesis, 1998, University of Auckland, New Zealand.

[32] Hunter, P., Nielsen, P., Smaill, B., LeGrice, I. and Hunter, I. An anatomical heart model with applications to myocardial activation and ventricular mechanics, Crit Rev Biomed Eng, 1992, 20, 403-426.

[33] Ogden, R. Non-linear elastic Deformations, 1997, Dover Publications, New York.

[34] Mooney, M. A theory of large elastic deformation, Journal of Applied Physics, 1940, 11, 582-592.

[35] Pathmanathan, P. Predicting tumour location by simulating the deformation of the breast using nonlinear elasticity and the finite element method, D.Phil Thesis, 2007, University of Oxford. 
[36] Saad, Y. and Schultz, M. GMRES: A generalized minimal residual algorithm for solving nonsymmetric linear systems, SIAM Journal on Scientific Computing, 1986, 7, 856-869.

[37] Whiteley, J., Bishop, M., and Gavaghan, D. Soft tissue modelling of cardiac fibres for use in coupled mechanoelectric simulations. Bull. Math. Biol., 2007, 69, 2199-2225.

[38] Kloosterman, G. Contact Methods in Finite Element Simulations, PhD thesis, 2002, Universiteit Twente, The Netherlands.

[39] Houston, P., Schötzau, D. and Wihler, T. An $h p$-adaptive mixed discontinuous Galerkin FEM for nearly incompressible linear elasticity, Computer Methods in Applied Mechanics and Engineering, 2006, 195, 32243246.

[40] Ten Eyck, A. and Lew, A. Discontinuous Galerkin methods for nonlinear elasticity. International Journal for Numerical Methods in Engineering, 2006, 67, 1204-1243.

[41] Sukumar N., Moës, N., Moran, B. and Belytschko, T. Extended finite element method for three-dimensional crack modeling. International Journal for Numerical Methods in Engineering, 2000, 48(11), 1549-1570. 


\section{List of Figures}

1 Simulating the compression of the breast during mammography. Note that only front (skin, lighter coloured) and back (pectoral muscle, darker coloured) surfaces of the breast are shown. Also shown are the compression plates. (a) The initial state before compression. (b) The computed compressed breast. (c) Alternative view of the compressed state. . . . . . . . . . .

2 The elements used in the numerical schemes, together with the number of unknowns which have to be computed, illustrated using either the pressure, $p$, or the displacement in the $X$-direction, $u$. Note that different combinations of these 6 elements are used in each of the 4 schemes (so that the Linear method would use the top-right element for $u$ and for $v$ (the two components of displacement), and the top-left element for $p$ ). Top row: piecewise constant quadrilateral (used in the linear scheme); linear triangle (used for the pressure in the quad-tri scheme); (bi-)linear quadrilateral (used for displacement in the linear scheme and pressure in the quad-quad and cubic-Hermite schemes). Bottom row: quadratic triangle (used for displacement in the quad-tri scheme); (bi-)quadratic quadrilateral (used for displacement in the quad-quad scheme); cubic-Hermite quadrilateral (where the given derivatives of the unknown also have to be computed at

each node). . . . . . . . . . . . . . . . . 
Deformed shapes for each of the model problems (solid lines), with undeformed unit square (dashed lines). (a): Model Problem 1a, where the body is stretched in the $X$-direction and compressed in the $Y$-direction (more stretching/compression as $X$ increases). Model Problem 1b is a $3 \mathrm{D}$ variation on this. (b): Model Problem 2, where the body has discontinuous material properties and the solution is one shear with gradient $-\alpha$ in $X \leq 0.5$, and a second shear with gradient $-\beta$ in $X>0.5$. (c): Model Problem 3 , where the solution is near zero displacement for $X<0.5$, a sharp compression in the $X$-direction (hence elongation in the $Y$ direction) around $X=0.5$, and a translation inwards for $X>0.5$. (d): Model Problem 4, where the bottom surface is fixed, and a discontinuous force is applied to the top surface: zero traction except in the region $\{X \in[0.25,0.75], Y=1\}$. Note that in Model Problems 1-3, only the left-hand edge is fixed, surface tractions are applied to the remaining three edges. . . . . . . . . . . 12

4 Results with Model Problem 1a: error plotted against spatial stepsize, using the: linear method (solid lines with stars); quadtri (dotted lines with triangles); quad-quad method (dashed lines with diamonds); and cubic-Hermite method (dot-dashed lines with squares). . . . . . . . . . . . . . . . . . 20

$5 \quad$ Results with Model Problem 1b (3D): error plotted against spatial stepsize, using the: linear method (solid lines with stars); quad-tri (dotted lines with triangles); quad-quad method (dashed lines with diamonds); and cubic-Hermite method (dot-dashed lines with squares). . . . . . . . . . . . . . 22 
Results with Model Problem 2, error plotted against spatial stepsize. The two figures plot the same results except the linear method is not plotted in (b). Methods plotted are: the linear method (solid lines with stars); quad-tri (dotted lines with triangles); quad-quad method (dashed lines with diamonds); and cubic-Hermite method (dot-dashed lines with squares). . . . . . .

7 The location of the deformed nodes when the quad-tri method is used on Model Problem 2 with $h=1 / 16$. (Note that internal nodes are shown as well as vertices, which is why there are $33^{2}$ nodes rather than $\left.17^{2}\right) \ldots \ldots \ldots \ldots \ldots$

8 Results with Model Problem 3, error plotted against (a) spatial stepsize and (b) number of degrees of freedom. Methods plotted are: the linear method (solid lines with stars); quad-tri (dotted lines with triangles); quad-quad method (dashed lines with diamonds); and cubic-Hermite method (dot-dashed lines with

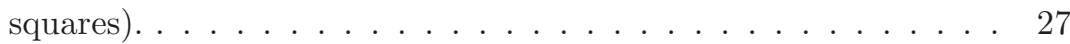

9 Results with Model Problem 4, the normal stress (scaled by $c$ ) on the top surface of the body. (a) The linear method (solid line) and quad-tri method (dashed line). (b) The quad-quad method (solid line) and cubic-Hermite method (dashed line). The dotted lines are just the lines $y=-3 \ldots \ldots \ldots \ldots$

10 Results with Model Problem 4: (a) $y(0.5,1)$ plotted against spatial stepsize and (b) the average error in $S^{22} / c$ against stepsize. Methods plotted are: the linear method (solid lines with stars); quad-tri (dotted lines with triangles); quad-quad method (dashed lines with diamonds); and cubic-Hermite method (dot-dashed lines with squares) (not plotted in (b)). . . . . . . . . . 30 



\section{RECENT REPORTS}

2009

01/09 A Mass and Solute Balance Model for Tear Volume and Osmolar- Gaffney ity in The Normal And The Dry Eye Tiffany

Yokoi

Bron

02/09 Diffusion and permeation in binary solutions Peppin

03/09 On the modelling of biological patterns with mechanochemical Moreo models: insights from analysis and computation Gaffney

Garcia-Aznar

Doblare

04/09 Stability analysis of reaction-diffusion systems with timem- Madzvamuse dependent coefficients on growing domains

Gaffney

Maini

05/09 Onsager reciprocity in premelting solids

Peppin

Spannuth

Wettlaufer

06/09 Inherent noise can facilitate coherence in collective swarm motion

Yates et al.

07/09 Solving the Coupled System Improves Computational Efficiency of the Bidomain Equations

Southern

Plank

Vigmond

Whiteley

08/09 Model reduction using a posteriori analysis

Whiteley

09/09 Equilibrium Order Parameters of Liquid Crystals in the LaudauDe Gennes Theory

10/09 Landau-De Gennes theory of nematic liquid crystals: the OseenFrank limit and beyond

Majumdar

Majumdar

Zarnescu

11/09 A Comparison of Numerical Methods used for Finite Element Modelling of Soft Tissue Deformation

Pathmanathan

Gavaghan

Whiteley

12/09 From Individual to Collective Behaviour of Unicellular Organisms: Recent Results and Open Problems

Xue

Othmer

Erban

13/09 Stochastic modelling of reaction-diffusion processes: algorithms Erban for bimolecular reactions

Chapman

14/09 Chaste: a test-driven approach to software development for phys-

Pitt-Francis et al. iological modelling 
15/09 Block triangular preconditioners for PDE constrained optimiza- Rees tion Stoll

16/09 From microscopic to macroscopic descriptions of cell migration on Baker growing domains Yates

Erban

Copies of these, and any other OCCAM reports can be obtained from:

Oxford Centre for Collaborative Applied Mathematics Mathematical Institute

24 - 29 St Giles'

Oxford

OX1 3LB

England

www.maths.ox.ac.uk/occam 